\title{
Diffuse parenchymal neurocysticercosis
}

\author{
S Ankara, K Dhungel, K Ahmad, M K Gupta, A Panwar \\ Department of Radiodiagnosis and Imaging \\ B.P. Koirala Institute of Health Sciences, Dharan, Nepal
}

\begin{abstract}
Neurocysticercosis is the commonest parasitic manifestation of brain and the leading cause of seizure in the developing world. It is caused by the ingestion of Taenia solium eggs through contaminated food and especially pork meat. Computed tomography and magnetic resonance imaging are the modality of choice for diagnosis of neurocysticercosis. We report a case of 65 years old male presented with complaint of seizure, which was diagnosed as diffuse parenchymal neurocysticercosis on computed tomography scan of brain.
\end{abstract}

Keywords: Computed tomography, neurocysticercosis, taenia solium

\section{Introduction}

Encystations of the larvae of Taenia solium (pork tapeworm) in the tissues is known as cysticercosis. Encystation of this pork tapeworm in central nervous system is called neurocysticercosis (NCC) and is endemic in developing countries. It is considered as the commonest parasitic manifestation of the central nervous system leading to seizure in the developing world. ${ }^{1,2}$ Clinically it can manifests as epilepsy, intracranial hypertension, focal neurological signs, cerebellar ataxia, cognitive decline, symptoms of hydrocephalus and psychiatric

\footnotetext{
Address for correspondence

Dr. Sajid Ansari

Assistant Professor

Department of Radio Diagnosis and Imaging

B.P. Koirala Institute of Health Sciences, Dharan

Email: drsajidansari@yahoo.co.in
}

disorders. The clinical manifestations vary with the number, size, location and stage of cysticerci as well as the intensity of the host's immune response. ${ }^{3}$

\section{Case report}

A 65 years old male presented with multiple episodes of generalized tonic-clonic seizure. There was no family history of epilepsy. No history of trauma. Physical examination was normal and showed no neurologic deficits. Laboratory tests revealed mild leukocytosis. Computed Tomographic (CT) scan of brain was done. Axial section non-enhanced followed by contrast-enhanced CT of the brain revealed multiple, small, rounded, well defined hypodense lesions diffusely scattered in brain parenchyma involving bilateral 
fronto-temporo-occipito-parietal lobes and bilateral cerebellar hemispheres (Figure 1, 2, 3 and 4).

Figure 1, 2, 3 and 4: Axial section CT image of brain showing multiple, small, rounded, well defined hypodense lesions diffusely

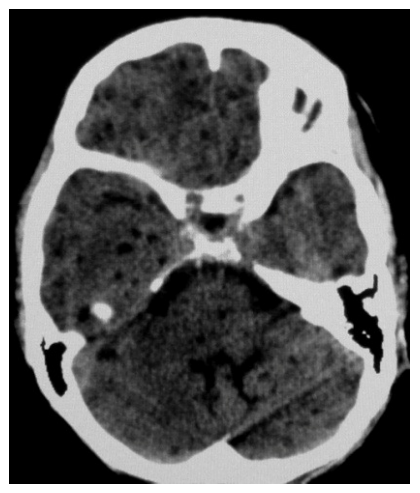

Fig. 1

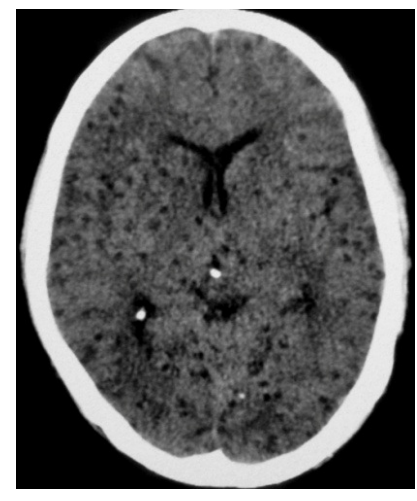

Fig. 3

Lesions were also seen in pons, midbrain, thalamus, basal ganglia and corpus callosum. Calcified mural nodules are seen within some of the lesions, suggesting calcified scolex (Figure 2, 3 and 4) and mild perilesional edema in some of the lesions. There was no scattered in brain parenchyma involving bilateral fronto-temporo-occipito-parietal lobes and bilateral cerebellar hemispheres. Calcified mural nodules within some of the lesions, suggesting calcified scolex seen in left occipital and right parietal.

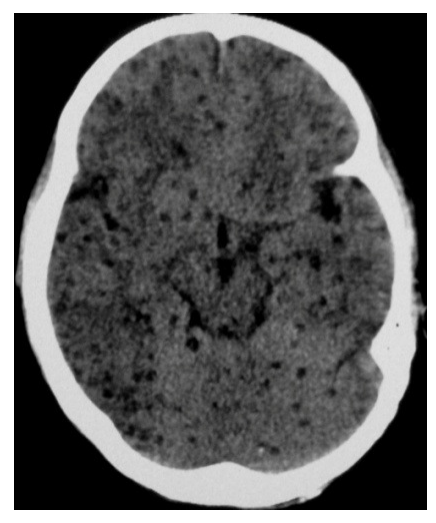

Fig. 2

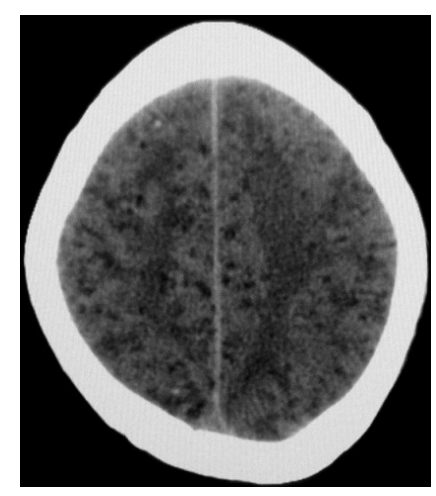

Fig. 4

evidence of hydrocephalus. The patient was managed conservatively with albendazole, dexamethasone and phenytoin. At 3-month follow-up, there was no seizure and the patient was on phenytoin. 


\section{Discussion}

Ingestion of Taenia solium eggs (pork tapeworm) through contaminated food results in cysticercosis. ${ }^{4}$ When the eggs reach the intestine, their protective coatings are destroyed by biliary and pancreatic enzymes and released as oncospheres. Then they traverse the intestinal wall and enter the bloodstream and are carried to the tissues of the host and established as larvae. The oncospheres lodged in the central nervous system develops into cysticercus cellulosae and known as NCC. ${ }^{4}$ This sets up an inflammatory reaction in the CNS which produces seizures and epilepsy. ${ }^{2}$

Neurocysticercosis is further divided into parenchymal and extraparenchymal disease. The CSF (cerebrospinal fluid) migration of cysticerci to the ventricles, cisterns and subarachnoid space or within the eyes or spinal cord is called extraparenchymal disease. The intraventricular and basal cisternal NCC along with hydrocephalus has mortality rate of about $50 \%{ }^{3}$

Patients with cysticercosis are typically aged 10-40 years. However, cases have been described in every age group. There is no gender predilection. ${ }^{3}$ Postmortem studies in endemic areas suggest that $80 \%$ of neurocysticercal infections are asymptomatic. $^{6}$ Consequently, many cases are never diagnosed or are found incidentally during imaging procedures.
According to Escobar classification, NCC is categorized into four stages. ${ }^{7}$ Stage 1 (vesicular stage) is the active or viable stage. CT scan shows round, hypodense areas of varying in sizes and numbers, with hyperdense eccentric mural nodule within (2$4 \mathrm{~mm}$ in size) and no perilesional edema. No evidence of enhancement on contrast study. On MRI (magnetic resonance imaging), it appears as CSF-like intensity signal on all sequences with hyperintense mural nodule within.

Stage 2 (colloidal-vesicular stage) is the transitional stage. The cysticercus loses the ability to control the host's immune response and the cyst wall becomes infiltrated and is surrounded by predominantly mononuclear cells. On CT and MRI, it appears as ring enhancing lesions with perilesional edema.

Stage 3 (granular-nodular stage) is also the transitional stage in which host's immune response further progresses with collapse of the cyst cavity. On CT, it appears as small, hyperdense, rounded, nodular lesion surrounded by edema. On MRI, it appears as signal void in all the sequences with perilesional edema.

Stage 4 (calcified nodular stage) is the inactive stage formed as a result of dystrophic calcification of the necrotic larva. It is seen as calcified nodule with no perilesional edema on $\mathrm{CT}$ and on $\mathrm{MRI}$, it is 
seen as signal void in all the sequences with no perilesional edema.

The treatment of NCC depends upon the viability of the cyst and its complications. Steroids, antihelminthics and antiepileptics are used for treating. The cysts in the active and transitional stage are treated with steroids. ${ }^{5}$ Complication of intraventricular NCC is hydrocephalus which is managed surgically by ventricular shunting followed by surgical extirpation of the cyst and subsequent medical treatment. In most patients with NCC, the prognosis is good. Patients with complications such as hydrocephalus, large cysts, multiple lesions with edema, chronic meningitis, and vasculitis are acutely ill and do not respond very well to treatment. There is no role for cysticidal drugs in inactive neurocysticercosis (stage 4) as the parasites are dead and given only symptomatic treatment. ${ }^{8}$

\section{Conclusion}

NCC is the commonest parasitic infection of brain and a leading cause of seizure in the developing world. CT and MRI are the best imaging modalities for diagnosing, staging and recurrence of NCC. Response to treatment can also be evaluated on neuroimaging.

\section{References}

1. Kraft R. Cysticercosis: An Emerging Parasitic Disease. Am Fam Physician 2007;75: 91-96.

2. DeGiorgio C M, Houston I, Oviedo S, Sorvillo F. Death associated with cysticercosis: Report of three cases and review of the literature. Neurosurg Focus. 2002; 12:1-4.

3. Takayanagui OM, Odashima NS. Clinical aspects of neurocysticercosis. Parasito Int 2006; 55:S111-5.

4. Jorge $G B$, lan $P$, Hector $H G$. Neurocysticercosis in a patient in Canada. CMAJ 2009; 180(6):639-642.

5. Garg R K. Drug treatment of neurocysticercosis. Natl Med J India. 1997;10:173-177.

6. Wallin MT, Kurtzke JF. Neurocysticercosis in the United States: review of an important emerging infection. Neurology 2004; 63(9):1559-64.

7. Escobar A. The pathology of neurocysticercosis of the central nervous system. In: Palacios E, RodriguezCarbajal J, Taveras JM, (Ed). Cysticercosis of the central nervous system. Springield, IL: Charles C Thomas 1983:27-54.

8. Bothale KA, Mahore SD, Maimoon SA. A rare case of disseminated cysticercosis. Trop Parasitol 2012; 2:138-141 